\title{
ESTUDIOS
}

\section{La responsabilidad social corporativa en las PYME: un estudio en empresas asturianas de economía social}

\section{María Leticia Santos Vijande, María Begoña González-Busto Múgica y Begoña López Fernández'}

Palabras Clave: Economía Social, Responsabilidad Social, PYME, Clientes, Proveedores, Información a la Sociedad, Colaboración Responsable,Asturias (España).

Key words: Social Economy, Corporate Social Responsibility, SME, Clients, Suppliers, Information to Society, Social Cooperation, Asturias (Spain).

En este trabajo, realizado entre empresas de Economía Social del Principado de Asturias, se ofrece una visión del tipo de prácticas socialmente responsables que desarrollan estas organizaciones en el ámbito externo o social de sus operaciones, es decir, en la relación mantenida con sus interlocutores comerciales más directos, clientes y proveedores, y con la sociedad en la que desarrollan su actividad. Este último aspecto se concreta en el estudio tanto de los procedimientos dirigidos a la información y comunicación con la sociedad, como de las fórmulas de colaboración responsable que acometen las empresas de Economía Social analizadas. Así se trata de contribuir a nuestra compresión de la trascendencia práctica de la Responsabilidad Social Corporativa (RSC) en la vida de las empresas, superando las aproximaciones más tradicionales llevadas a cabo desde perspectivas sociolaborales y medioambientales.

\footnotetext{
1 Profesoras Titulares de Universidad. Departamento de Administración de Empresas y Contabilidad. Facultad de Ciencias Económicas y Empresariales. Universidad de Oviedo. Texto final recibido el 24 de abril de 2008.
} 
Dado que el estudio se desarrolla en un contexto de PYME de Economía Social, permite aportar evidencias prácticas sobre los esfuerzos que desarrollan en materia de RSC no sólo las organizaciones más pequeñas, sino las que además cuentan con una clara vocación de servicio social. La investigación se completa con el análisis de dos aspectos adicionales: a) los objetivos perseguidos por las empresas con el desarrollo de las prácticas de RSC, para determinar si los beneficios esperados superan un enfoque utilitarista centrado en los resultados económicos; y b) el papel atribuido a las Administraciones Públicas en materia de RSC, para evaluar si la RSC se concibe como fruto de la voluntariedad moral o de la regulación.

Para alcanzar estos objetivos el trabajo se estructura como sigue: comenzamos analizando brevemente el concepto de RSC, a continuación se describen los beneficios que se pueden derivar de las prácticas socialmente responsables, incluso entre las empresas de menor tamaño, aunque estemos ante una actividad tradicionalmente atribuida y difundida por las grandes empresas y multinacionales; en tercer lugar se examina la aplicación de la RSC desde la perspectiva de las empresas de Economía Social, es decir, la correspondencia entre los principios que rigen la actividad de estas empresas y el comportamiento socialmente responsable; a continuación, se describen las características y resultados del estudio empírico, detallando las características de la muestra y las prácticas socialmente responsables ejecutadas; por último, acabamos con las principales conclusiones que se pueden extraer de este trabajo.

\section{La responsabilidad social corporativa}

Desde la segunda mitad del siglo XX en las economías de los países desarrollados se ha podido presenciar un cambio generalizado en el concepto de empresa basado en la concienciación progresiva de la responsabilidad social que deben asumir las entidades lucrativas y en la necesidad de superar la visión empresarial centrada en la maximización del beneficio a cualquier precio. Se avanza de este modo hacia una orientación menos economicista y más humanista del modelo capitalista liberal que obliga a las empresas a realizar cambios en sus estrategias empresariales para responder a las nuevas inquietudes y expectativas de los ciudadanos, consumidores, poderes públicos e inversores los cuales, en el contexto de la globalización y el cambio industrial, reclaman una mayor sensibilidad e inversión social de las organizaciones para contribuir al Estado del Bienestar (OLCESE, 2006).

La RSC es un término dinámico, que aún hoy en día se sigue refinando, y al que también se designa como Responsabilidad Social Empresarial (RSE), para alejar 
cualquier connotación estrictamente vinculada a las grandes empresas. Este concepto ha evolucionado desde la primera aproximación moderna atribuida a Bowen (1953), quien al referirse a los procesos de decisión de las empresas, argumenta que éstos deben considerar no sólo la dimensión económica, sino también las consecuencias sociales que se derivasen del comportamiento empresarial, hasta aproximaciones más recientes como la enunciada por el Consejo Empresarial Mundial para el Desarrollo Sostenible (1999)2. Para esta organización empresarial la RSC representa "el compromiso continuo de las empresas de comportarse éticamente y de contribuir al desarrollo económico, a la vez que mejoran la calidad de vida de los trabajadores y sus familias, de las comunidades locales y de la sociedad en general". Es decir, las empresas no sólo deben preocuparse por optimizar al máximo los beneficios y repartir dividendos, sino que también deben estar preocupadas por la forma en que los obtienen y por su contribución a la sociedad. De este modo, los administradores de las empresas deben responder no sólo ante los accionistas sino también ante cualesquiera otros colectivos humanos implicados en las actuaciones de la empresa. Estos grupos de interés, denominados "stakeholders" incluyen, entre otros, a trabajadores, clientes, proveedores, sindicatos, Administraciones Públicas, grupos ecologistas y, en general, a toda la comunidad en la que opera la empresa.

El Libro Verde sobre la RSC elaborado por la Comisión Europea (2001) refuerza la definición previa diseñada del mundo empresarial destacando, además, la voluntariedad de las prácticas socialmente responsables: "integración voluntaria, por parte de las empresas, de las preocupaciones sociales y medioambientales en sus operaciones comerciales y sus relaciones con los interlocutores". En este mismo sentido, el informe final elaborado en nuestro país por el Foro de Expertos en RSE3 (2007) establece que "la RSC es, además del cumplimiento estricto de las obligaciones legales vigentes, la integración voluntaria en su gobierno y gestión, en su estrategia, políticas y procedimientos, de preocupaciones sociales, laborales, medio ambientales y de respeto a los derechos humanos...".

${ }^{2}$ El Consejo Empresarial Mundial para el Desarrollo Sostenible, (World Business Council for Sustainable Development), también conocido por sus siglas WBCSD, es una coalición de compañías internacionales comprometidas en pro del desarrollo sostenible mediante el crecimiento económico, el equilibrio ecológico y el progreso social. Este organismo fue creado en 1995 y, en la actualidad, cuenta con más de 200 empresas asociadas: www.wbcsd.org.

${ }^{3}$ Foro constituido el 17 de marzo de 2005 por iniciativa del Ministerio de Trabajo y Asuntos Sociales con la participación de representantes de varios Ministerios y de expertos provenientes de grupos empresariales, organizaciones de la sociedad civil y de la Universidad. Concluye su trabajo en julio de 2007. 
En definitiva, desde una perspectiva socialmente responsable se requiere que las organizaciones, voluntariamente y hasta más allá de lo legalmente exigible, busquen cubrir las expectativas económicas, legales, éticas y sociales de todos los individuos que afectan o pueden ser afectados por el logro de sus objetivos.

Estamos, pues, ante una nueva forma de ser empresa en la sociedad, una nueva cultura empresarial, que tiene que ver con una empresa que convive respetuosamente con las personas y con el entorno. No es posible reducirlo a un nuevo marketing social o a la dedicación de parte de los beneficios para la acción social. Esto, si se da, será una parte de la RSC, pero no será su elemento constitutivo (CASTRO SANZ, 2005, 2006). Por otra parte, la RSC constituye una cultura empresarial muy acorde con los objetivos definidos en la Estrategia de Lisboa aprobada en el año 2000 , en la que la UE se propuso convertirse para el año 2010 "en la economía basada en el conocimiento más competitiva y dinámica del mundo, capaz de crecer económicamente de manera sostenible con más y mejores empleos y mayor cohesión social". Queda claro que la estrategia de la UE incluye, por tanto, aliar el crecimiento económico con la cohesión social, afirmando que otra forma de crecer es posible, por lo que otra forma de ser empresa es posible.

El concepto de RSC está además íntimamente unido a los tres principios que guían el desarrollo sostenible: a) la integridad medioambiental; b) la prosperidad económica; y c) la igualdad social (EIKINGTON, 1997). Así pues, se puede decir que la RSC incluye tres procesos clave: 1) la evaluación medioambiental; 2) la gestión de los grupos de interés o "stakeholders" como base para garantizar la prosperidad económica, y 3) la gestión de las cuestiones sociales (WOOD, 1991).

Desde esta perspectiva, las relaciones de la empresa con su entorno en el ámbito de la RSC incluyen una dimensión interna, una dimensión externa y una dimensión medioambiental. En la dimensión interna, aquella que tiene que ver con los procesos y actividades que realiza de puertas adentro una empresa, cabe incluir dos grandes bloques de actuaciones:

- La relación de los accionistas con los directivos. Las ineficiencias asociadas a esta relación ha dado lugar a un amplio debate en las sociedades occidentales sobre cómo se deben gobernar las organizaciones, lo que se ha venido a denominar "Gobierno Corporativo". Así, desde la década de los 90 se han elaborado Códigos (códigos de Buen Gobierno) que, desde el ámbito de la voluntariedad, dan recomendaciones a las empresas en materias como relaciones con los accionistas, prácticas anticorrupción, transparencia informativa, etc., con el objetivo de mejorar las estructuras de gobierno empresarial. 
- Aspectos sociales de la propia actividad de la empresa con sus trabajadores y empleados que incluyen, entre otros, aspectos como la salud y bienestar de los trabajadores, la formación, la participación, la conciliación de la vida familiar y laboral, la igualdad y la atención a colectivos desfavorecidos.

La dimensión externa de la RSC engloba las relaciones de la empresa con aquellos grupos de interés con los que interacciona fuera de su ámbito interno, estos son, entre otros, los proveedores, clientes, consumidores y usuarios, inversores, el mercado de capitales, el Gobierno y las Administraciones y, en general, la comunidad en la que la empresa desarrolla su actividad.

La tercera dimensión que constituye un aspecto diferenciado de la RSC es la medioambiental. La RSC medioambiental comprende las actividades cuyo objeto es la reducción del impacto medioambiental de la empresa, a través, entre otros, de la reducción y control de consumos y residuos o los sistemas de gestión medioambiental.

En resumen, la RSC implica una manera de ver la empresa y de hacer negocios, desde el compromiso con el desarrollo sostenible, la responsabilidad hacia todos los grupos de interés o "stakeholders" e íntimamente ligada a la ética empresarial y a la transparencia.

\section{La Responsabilidad Social Corporativa en las PYME}

Es cierto que hoy en día la importancia de la RSC es creciente y que cada vez más empresas se preocupan por esta forma de gestión empresarial por la que se comprometen a actuar de forma correcta en la consecución de sus objetivos. Pero no es menos cierto que frecuentemente al hablar de RSC la referencia son las grandes empresas. Sin embargo, en la Unión Europea, las pequeñas y medianas empresas representan el 99,9\% de los casi 19,3 millones de empresas existentes y generan el $66 \%$ del empleo privado. En nuestro país, de acuerdo con el Directorio Central de Empresas (DIRCE) a 1 de enero del año 2006 había en España 3.161.480 PYME (empresas comprendidas entre 0 y 249 asalariados). Es decir, el 99,87 por ciento de las 3.165.619, empresas que conforman el censo, excluida la agricultura y la pesca.

Las PYME juegan, por tanto, un papel muy importante en el tejido empresarial español y también europeo, y aunque son fuente de crecimiento, no es menos cierto que encuentran, dado su menor tamaño y falta de recursos económicos y humanos, 
dificultades para aplicar o avanzar en el ámbito de la RSC. Así entre las barreras internas con las que se encuentran las PYME en el ámbito de la responsabilidad social se pueden mencionar la falta de información, formación y capacitación en temas de sostenibilidad, que hacen que las empresas sólo perciban la RSC como una fuente de costes y no de competitividad; habilidades técnicas y de gestión limitadas en algunos campos; insuficiente conocimiento de la legislación, o la falta de recursos para construir relaciones externas y redes de comunicación de las estrategias sostenibles. Para las PYME resulta claro que las empresas grandes están necesitadas de logos y frases "que las unan a la sociedad", y más si cotizan en Bolsa, pero que estos símbolos no repercuten de forma tan automática en el caso de las PYME cuyo nombre no sale todos los días en los medios, y que por tanto "existen en menor medida" para el consumidor medio. Igualmente, existen barreras económicas como la mayor dificultad de las PYME para conseguir economías de escala; su comportamiento menos competitivo en algunos mercados; su mayor dificultad para obtener financiación y el mayor coste de capital que soportan (Salas y García-Orcoyen, 2006; Vázquez, 2006).

Sin embargo, y pese a estas dificultades, la literatura ofrece distintos argumentos a favor de la implantación de la RSC en las PYME, los cuales coinciden con los beneficios que, con carácter general, se pueden esperar de las prácticas socialmente responsables:

- La RSC permite mejorar las relaciones con los distintos grupos de interés, lo que aumenta la reputación de las empresas y permite un mejor acceso a las ayudas públicas.

- La RSC ayuda además a las PYME a desarrollar nuevas formas de trabajar, más orientadas al mercado, lo que permite gestionar mejor los riesgos e identificar oportunidades.

- La mayor atención a las necesidades de los trabajadores comporta la reducción del absentismo laboral, el aumento de la productividad y la mayor motivación y fidelidad de los trabajadores, lo que en redunda en mejoras en la innovación y en la propia calidad de los bienes y servicios ofertados (CANALS Y FONTRODONA, 2006).

- Este hecho favorece un mejor alineamiento de las empresas con las necesidades de los consumidores, genera mayor lealtad y satisfacción y permite mantener las relaciones comerciales a largo plazo. 
- También se producen ahorros en costes e incremento de la rentabilidad a medio plazo, debido a la mayor eficiencia en el uso de los recursos humanos y productivos y a la mejora de la posición comercial.

Además, es necesario considerar que muchas PYME, más que perseguir la maximización de beneficios, persiguen alcanzar una rentabilidad satisfactoria que les permita garantizar su viabilidad junto con otros objetivos sociales o morales, considerándose además extremadamente importantes las relaciones humanas que se establecen tanto en el seno de la empresa como entre la empresa y otros grupos de interés, como clientes y proveedores (VIVES, CORRAL E ISASI, 2005). En este contexto la RSC constituye un instrumento de innegable valor.

\section{La RSC desde la perspectiva de las empresas de Economía Social}

La Economía Social significa una específica forma de hacer empresas, de generar empleo y crear riqueza colectiva que no sólo permite hacer frente a las necesidades materiales sino que, al mismo tiempo, permite colaborar en la configuración de un mundo más confortable, más solidario y más humano (PINO, 2003). Así, las empresas de Economía Social se rigen por los denominados Principios Cooperativos ${ }^{4}$ (MILÁN Y BERINI, 2006) los cuales se inspiran en los valores de autoayuda, auto-responsabilidad, democracia, igualdad, equidad y solidaridad. Las empresas de Economía Social se ven así dotadas de una personalidad especial que las sitúa en una posición preferente ante la RSC. De hecho, varios principios del Pacto Mundial referidos a la RSC muestran una clara correspondencia con los Principios Cooperativos establecidos en 1844 y adaptados en septiembre de 1995 por la Alianza Cooperativa Internacional (COLLADO, 2006).

Hablamos, en definitiva, de gestión económica y acción empresarial, pero realizada desde valores y objetivos apoyados en la cohesión y solidaridad social, en la trans-

\footnotetext{
${ }^{4}$ Los principios cooperativos, son los elementos distintivos de las organizaciones y empresas cooperativas y, por extensión de las empresas de Economía Social. Se han ido reformulado a lo largo de más 150 años para adaptarlos a la época histórica actual, manteniendo el núcleo de un sistema que los Pioneros de Rochadle, fundadores en 1844 de la primera cooperativa de la historia entendida como tal, formularon de forma simple y clara para asegurar el funcionamiento de la sociedad cooperativa. En la actualidad los Principios Cooperativos son los siguientes: (1) asociación voluntaria y abierta; (2) control democrático por los asociados; participación económica de los socios; (3) autonomía e independencia; (4) educación, capacitación e información; (5) cooperación entre cooperativas y preocupación por la comunidad.
} 
parencia y en la equidad para distribuir las rentas. La Economía Social, además, significa la gestión participativa situando a la persona por encima del capital, el cual se entiende como un instrumento; necesario, pero tan solo un instrumento. Este modo de proceder en empresas que actúan en el mercado en condiciones de competencia, significa que es posible otra forma de hacer empresa, representativa por añadidura de valores cercanos al sentir social (CASTRO Sanz, 2005, 2006).

El "Informe de la Subcomisión del Congreso para potenciar y promover la Responsabilidad Social de la empresas" (Boletín Oficial de las Cortes Generales de 31 de julio de 2006), reconoce que las empresas de Economía Social tienen características que las diferencian del resto y que las sitúan en una tradición de clara sintonía con la intuición de fondo de la RSC: la posibilidad de simultanear la viabilidad económica y la responsabilidad social. De hecho, el informe cita la comparecencia de la Confederación Empresarial Española de Economía Social (CEPES) ante la Subcomisión y los argumentos que este organismo esgrime acerca de las aportaciones de la Economía Social al comportamiento empresarial socialmente responsable:

- Creación de empleo, las empresas de Economía Social crecen normalmente cuatro puntos porcentuales por encima de lo que crece la población ocupada, creando empleo estable en mayores proporciones que el generado por el mercado.

- Regulación y equilibrio tanto de sectores económicos (economía agraria, por ejemplo) como sociales (enraizando a las personas en sus territorios naturales dado que estas empresas son ajenas al fenómeno de la deslocalización, por lo que se evitan flujos migratorios).

- Generación de riqueza allá donde el inversor tradicional no tiene motivaciones para existir (yacimientos de empleo, por ejemplo).

- Desarrollo del espíritu emprendedor (los índices de creación de empresas en estas formas jurídicas son muy elevados).

- Activación de la participación en la gestión económica ("escuelas de democracia económica") y formación para la gestión empresarial.

- Creación de empresas para cubrir nuevas necesidades sociales.

- Inclusión e inserción laboral de colectivos de difícil empleabilidad. 
A la luz de todas estas consideraciones, este trabajo analiza las prácticas socialmente responsables desarrolladas por empresas asturianas de Economía Social en un ámbito concreto: el referido a sus relaciones con los agentes externos. El objetivo es aportar evidencias empíricas acerca de si estas empresas responden efectivamente a sus objetivos sociales en el ámbito de estudio $y$, por añadidura, ilustrar el tipo de esfuerzos que desarrollan en la práctica las organizaciones que se caracterizan por su reducido tamaño.

\section{Estudio empírico sobre las prácticas de RSC en las empresas de Economía Social asturianas}

En este apartado se describe la "puesta en práctica de la investigación" o desarrollo del trabajo de campo llevado a cabo en este estudio. Se incluyen en primer lugar los procedimientos seguidos para el envío y seguimiento del cuestionario, la tabulación y el tratamiento estadístico la información recabada. También se comentan las características de la muestra de acuerdo con diversas variables de clasificación y los resultados del estudio. De modo específico, y tal y como se apunta en la introducción de ese trabajo, el estudio empírico buscar satisfacer tres objetivos:

- Identificar las motivaciones o beneficios que las organizaciones esperan de la aplicación de la RSC.

- Conocer el tipo de actividades concretas de RSC ejecutadas con mayor frecuencia. Las empresas se enfrentan a la posibilidad de desarrollar múltiples acciones de RSC, por lo que en este trabajo se plantea una selección de las mismas centradas en el comportamiento socialmente responsable de tipo externo o social. Éste se estudia en tres bloques atendiendo a si se refiere a las relaciones con clientes y proveedores, a los procedimientos de información y comunicación a la sociedad o a las fórmulas de colaboración responsable.

- Conocer si las empresas consideran necesario y/o urgente el desarrollo de iniciativas legislativas en materia reguladora de la RSC.

\section{I. Desarrollo de la investigación: trabajo de campo}

La recogida de información se llevó a cabo mediante un cuestionario estructurado -compuesto fundamentalmente por preguntas cerradas- cuya elaboración se fun- 
damentó en la revisión bibliográfica. Como paso previo a su redacción definitiva, este instrumento de medida fue sometido a un pre-test con el fin de detectar posibles inconsistencias o inconvenientes para su cumplimentación.

Se administró mediante un envío por correo postal dirigido al censo de empresas considerado en la investigación: empresas de Economía Social que operan en la zona Central de Asturias. Dicho censo fue facilitado por la patronal de las empresas de Economía Social en el Principado de Asturias, ASATA ${ }^{5}$ (Agrupación de Sociedades Asturianas de Trabajo Asociado) y, con el fin de incentivar la respuesta, fue acompañado de una carta explicativa de los objetivos del estudio elaborada desde la Agrupación.

Tras varios reenvíos, se obtuvieron un total de 39 encuestas debidamente cumplimentadas. La muestra analizada, por tanto, se corresponde con una tasa de respuesta de prácticamente el 10,0\%, como es habitual en este tipo de estudios. Cabe señalar que si bien el número total de cuestionarios debidamente cumplimentados no es elevado, resulta altamente satisfactorio, tanto desde la perspectiva del esfuerzo que supone para las empresas contestar a una encuesta tan detallada sobre RSC, como por el índice de respuesta que representa. Los encuestados ocupan los niveles más altos de dirección en el 69,2\% de las respuestas (gerente, presidente o directores de departamento), lo cual acredita la calidad de la información recogida.

\subsection{Características de las empresas analizadas}

En este epígrafe se describen las características de las 39 empresas que constituyen la muestra. Para ello se analizan variables referidas a la forma jurídica y edad de las empresas, su ámbito de actividad y tamaño empresarial, medido en términos de número de trabajadores, el tramo de facturación en el que se sitúan, la amplitud de sus mercados y si disponen o no de certificaciones.

Los resultados muestran que la mayoría de las empresas analizadas $(51,3 \%)$ son Sociedades Anónimas Laborales, las Cooperativas representan el 30,8\% de las entidades y el 17,9\% restante esta formado por Sociedades Laborales Limitadas. La distribución muestral de la forma jurídica refleja la estructura poblacional ya que, de las 876 empresas de Economía Social que a finales de 2007 están activas

${ }^{5}$ La financiación para el desarrollo de la investigación ha sido proporcionada por la Agencia Local de Promoción Económica y Empleo del Ayuntamiento de Gijón. 
en la región ${ }^{6}$, el $62,7 \%$ son Sociedades Laborales y el resto cooperativas $(37,3 \%)$. Las empresas de la muestra son, en general, entidades jóvenes. Así, en conjunto, el $61,1 \%$ de las organizaciones han sido fundadas desde 1995 hasta la actualidad, lo que refleja el dinamismo de la Economía Social y su capacidad de atraer emprendedores. Aunque en el conjunto del territorio nacional el ritmo de crecimiento de la Economía Social se ha ralentizado en la última década, el Principado de Asturias ha acusado este hecho de manera menos marcada, manteniendo tasas de creación de empresas similares a las de la década de los noventa (FFES, 2003).

El 76,9\% de las empresas analizadas desarrollan su actividad principal esencialmente en el sector servicios; el 23,1\% restante lo hacen en la producción de bienes tangibles. Estos datos responden fielmente a la creciente terciarización de las economías de los países desarrollados. Así mismo, en la región el 72,8\% de las Cooperativas y el 63,0\% de las Sociedades Laborales operan en el sector servicios (FFES, 2007).

Para medir el tamaño empresarial se tomó como referente el número de trabajadores de acuerdo con la clasificación establecida por la Comisión de la Unión Europea ${ }^{7}$. La distribución de la muestra bajo este criterio pone de manifiesto que está compuesta exclusivamente por microempresas (menos de 10 trabajadores, $67,6 \%$ ) y pequeñas empresas (entre 10 y 49,32,4\%). De nuevo estos datos responden al perfil de conjunto de la región, la cifra media de trabajadores por empresa en estas entidades es de 9 individuos (FFES, 2007).

En cuanto al volumen de facturación el $61,6 \%$ de las empresas no supera los 300 mil euros anuales y sólo en el 12,8\% se sobrepasan los 600 mil. No obstante, los datos también muestran que, como cabría esperar, a medida que asciende el tamaño de las organizaciones en términos de empleo también crecen los volúmenes de facturación. Los últimos datos regionales disponibles en esta materia aportan cifras similares, $56,4 \%$ y $13,1 \%$ en cada tramo de facturación, respectivamente (FFES, 2003).

El ámbito geográfico del mercado en el que operan las empresas constó inicialmente de 4 categorías: local, regional, nacional e internacional. Finalmente se agruparon

\footnotetext{
${ }^{6}$ Los datos regionales actualizados están disponibles en la página web de la Fundación para el Fomento de la Economía Social: www.ffes.org.
}

\footnotetext{
${ }^{7}$ Así, se consideran microempresas a aquellas con menos de 10 trabajadores, empresas pequeñas las que tienen entre 10 y 49 , empresas medianas las que disponen entre 50 y 249 y grandes empresas las de más de 250 trabajadores.
} 
las dos últimas categorías con la finalidad de integrar a las pocas empresas que desarrollan su actividad internacionalmente. Así se puso de manifiesto que en la muestra existe un claro predominio de las empresas que atienden mercados regionales $(56,4 \%)$, seguidas de las que operan en mercados nacionales e internacionales $(23,1 \%)$ y locales $(20,5 \%)$.

La última variable de clasificación hace referencia a la disponibilidad de certificaciones, es decir, a la acreditación por organismos independientes de que las empresas desarrollan su actividad de acuerdo con estándares internacionales de calidad. Sólo el 15,8\% de las empresas analizadas cuentan con una certificación de su sistema de gestión de acuerdo con el estándar ISO 9000:2000. Este hecho no excluye que las correspondientes prácticas de calidad puedan estar siendo ejecutadas en las organizaciones sin que se haya solicitado la correspondiente certificación. De hecho, es reconocido que las empresas más pequeñas suelen encontrar en la certificación un incremento de su grado de formalización y documentación que les resulta complicado asumir. No obstante, la disponibilidad de este tipo de "sellos" de gestión suele utilizarse en los estudios sobre RSC como un indicador del interés por adoptar el mejor gobierno interno de las organizaciones posible.

\subsection{Motivaciones en el desarrollo de la RSC}

Al analizar el concepto de RSC se pone de manifiesto que el desarrollo de políticas socialmente responsables ofrece un abanico muy amplio de beneficios potenciales, que supera con creces los derivados de un comportamiento exclusivamente basado en el enfoque económico tradicional. En este apartado se estudian precisamente el tipo de motivaciones que consideran más determinantes para iniciar esta andadura. Es decir, se trata de establecer qué tipos de ventajas son percibidas por las organizaciones como consecuencias más probables de la RSC y que, por tanto, actúan como detonante de su adopción.

Para conseguir este objetivo fue preciso sintetizar el conjunto de posibles ventajas que debían valorar las organizaciones en un número razonable de opciones. Así se consideran tres boques de estímulos: a) ventajas de carácter económico; b) beneficios en el ámbito interno; y c) ventajas en el ámbito de social y mercado. Las variables analizadas en cada grupo se valoran en una escala de cinco posiciones, donde el valor 1 significa desacuerdo con la posibilidad de obtener el beneficio indicado (nada de acuerdo); 2, nivel reducido de acuerdo (algo de acuerdo); 3 acuerdo medio o moderado (de acuerdo); 4 acuerdo elevado (bastante de acuerdo); y 5, acuerdo absoluto (totalmente de acuerdo). 
En primer lugar se estiman las motivaciones asociadas al logro de ventajas de carácter económico, es decir, de aquellas que permiten la obtención de ventajas competitivas, o posiciones ventajosas frente a la competencia que afianzan la supervivencia empresarial a largo plazo. Así, las mejoras concretas en este ámbito vinculadas a la RSC fueron: la RSC permite facilitar la obtención de mayores beneficios, acceder más fácilmente a nuevos mercados, incrementar la cartera de clientes o acceder a incentivos fiscales.

El segundo bloque de contribuciones asociadas al desarrollo de la RSC explora sus efectos en el ámbito interno de las empresas. En el contexto actual, el compromiso e integración de los trabajadores es uno de los pilares fundamentales sobre el que apoyar la gestión empresarial para hacer frente a la flexibilidad y capacidad de adaptación que exigen los nuevos retos competitivos, así como para crear valor a los clientes. Por tanto, se trata de establecer si la RSC puede contribuir a mejorar el clima laboral, a aumentar el compromiso y participación de los socios y trabajadores de las empresas, y todo ello sin acrecentar la complejidad en la gestión.

El tercer bloque de motivaciones describe la eventual contribución de la RSC a la mejora de las relaciones tanto con el conjunto de la sociedad en general, como con los agentes del mercado en particular (clientes, proveedores y distribuidores), así como sus efectos en la imagen y prestigio de la empresa. Las organizaciones desarrollan una actividad social que afecta y se ve afectada no sólo por los interlocutores comerciales más directos, sino también por el conjunto de agentes sociales que interaccionan con la organización y cuyos intereses es necesario tener en cuenta. A su vez, la imagen y el prestigio corporativos son elementos que condicionan las posibilidades de negocio, puesto que sintetizan la apreciación externa de la actuación de las entidades. Por tanto, se trata de establecer si la RSC ayuda a mejorar las relaciones empresariales y a generar comunicación y confianza.

\subsection{Motivaciones de carácter económico}

Con respecto a la obtención de mayores beneficios como consecuencia de la aplicación de la RSC, las organizaciones de la muestra son bastante escépticas. Más de la mitad, el 53,8\%, está nada o muy ligeramente de acuerdo con este posible efecto y sólo un $10,3 \%$ se muestran bastante o completamente seguras respecto a la contribución de la RSC a la cuenta de resultados.

Las percepciones son muy similares cuando se valora la facilidad de acceso a nuevos mercados; tampoco parece que constituya un motivo lo suficientemente sólido como 
para interesar a la mayoría de las organizaciones analizadas que, en el $51,2 \%$ de los casos, apenas reconocen esta repercusión económica. El porcentaje de empresas muy convencidas se reduce al $7,8 \%$, mientras que el $41 \%$ mantienen una posición en la que atribuyen a la RSC un valor medio o moderado como mecanismo de acceso a nuevos mercados.

El incremento del número de clientes es un tipo de resultado que, sin embargo, las organizaciones consideran algo más probable como consecuencia de la aplicación de la RSC. Así, aunque sólo un 10,2\% de entidades están bastante o totalmente convencidas de este efecto, el porcentaje de empresas que están moderadamente de acuerdo con los efectos de la RSC en la ampliación de la cartera de clientes es el más elevado del conjunto de beneficios económicos (46,2\%). En consecuencia, se reconoce en mayor medida el potencial de la RSC para crear valor a los clientes de las organizaciones

En cuanto a los incentivos fiscales, estos se obtienen en la práctica de modo inmediato como resultado de ciertas actuaciones, por ejemplo, las donaciones a entidades no lucrativas. El 30,8\% de las empresas de la muestra parecen ser conscientes de este hecho puesto que indican que están bastante o totalmente convencidas de que la RSC les permitirá obtener ventajas fiscales. Sin embargo, en un ratio muy similar $(28,2 \%)$ las organizaciones no perciben este beneficio económico, mientras que el porcentaje de acuerdo moderado se sitúa en el $41 \%$.

Estos resultados se mantienen uniformes con independencia de la forma jurídica, tamaño, tipo de actividad, tipo de clientes atendidos, ámbito de mercado o volumen de facturación de las empresas de la muestra. De este modo, la principal conclusión que se puede extraer a partir de la consideración conjunta de los indicadores económicos es que estos constituyen mayormente un motivo secundario para las empresas a la hora de desarrollar la RSC.

\subsubsection{Motivaciones de ámbito interno}

El clima laboral alude al grado de satisfacción del conjunto de los integrantes de la empresa con el tipo de funcionamiento y gestión interna. En este ámbito parece que la probabilidad de obtener beneficios como consecuencia de la RSC se acrecienta notablemente. Así, casi 6 de cada 10 empresas analizadas $(56,4 \%)$ están bastante o totalmente de acuerdo en que el desarrollo de la RSC contribuye a mejorar el clima laboral. 
Este resultado en relación al clima laboral no se traslada completamente al incremento de la participación y compromiso de los trabajadores. De hecho, el porcentaje de empresas bastante o totalmente convencidas de este resultado decrece diez puntos $(46,1 \%)$, y un $36 \%$ de las empresas se muestran escépticas en cuanto a los posibles efectos de la RSC en la mayor involucración de los trabajadores (nada o algo de acuerdo).

Esto no quiere decir que el modelo de gestión de las empresas analizadas no goce de un alto nivel de compromiso y participación, sino que la RSC no se considera una fuerza tan determinante en esta circunstancia como en la mejora del clima laboral. De hecho, las empresas de Economía Social se caracterizan por ser más participativas como consecuencia de su estructura de propiedad.

El análisis de los efectos internos de la RSC se completa valorando la percepción acerca de sus implicaciones en la complejidad de la gestión. El 33,3\% de las empresas consideran que las políticas de RSC afectarán de modo inapreciable o reducido a la dificultad de la gestión organizativa, mientras que el $41 \%$, creen por el contrario que el impacto será muy significativo (bastante o totalmente). Este es un efecto adverso, que no sería deseable que fuese percibido por las empresas, pero que debe ser interpretado en clave del reducido tamaño de las organizaciones analizadas. Así, el desarrollo de nuevas iniciativas debe ser asumido por personas que, normalmente, desempeñan múltiples cometidos, por lo que es explicable que se asocie una mayor dificultad en la gestión a la puesta en práctica de la RSC.

En cualquier caso, es necesario tener en cuenta que las apreciaciones acerca de las motivaciones de carácter interno, o de los beneficios esperados de la RSC en el ámbito interno, resultan consistentes con independencia de las variables de clasificación consideradas.

\subsubsection{Motivaciones de carácter externo}

Prácticamente, el $75 \%$ de las empresas de la muestra consideran que la RSC es útil para mejorar sus relaciones con el entorno social. Sin embargo, este dato contrasta con la percepción que tienen las empresas acerca del interés de la comunidad en la que operan sobre la RSC. Más de un $50 \%$ la califican de reducida o muy reducida. Por tanto, aunque las empresas no se muestran muy de acuerdo con la existencia de una demanda social creciente de comportamiento socialmente responsable, sí que esperan obtener un rendimiento favorable en este ámbito como resultado de la aplicación de la RSC. 
Sin embargo, de acuerdo con los resultados, los principales beneficios o motivaciones que impulsan a las empresas analizadas se vinculan con la mejora de su imagen y prestigio.

La imagen de las organizaciones es una variable que cada vez resulta más importante en los mercados caracterizados por una fuerte competencia. Dicha imagen transmite de modo sintético la percepción colectiva de lo que la empresa es y hace $y$, bien gestionada, actúa como un claro elemento de diferenciación, reduce el riesgo de los clientes potenciales y facilita el acceso a nuevos mercados. Para 9 de cada 10 empresas de la muestra éste es un efecto esperable de la RSC $y$, además, 6 de cada 10 están bastante o totalmente de acuerdo con que se consigue este beneficio.

Del mismo modo, las políticas de RSC se perciben como instrumentos adecuados para fortalecer el prestigio empresarial o la valoración del mercado efectúa sobre el saber hacer de cada organización. De hecho, del conjunto de motivaciones consideradas, ésta es la que aglutina al porcentaje más elevado de empresas totalmente convencidas de que se trata de una consecuencia esperable de la RSC $(25,6 \%)$. No obstante, cabe destacar que las empresas escépticas en cuanto a la mejora del prestigio ascienden al 15,4\%, mientras que en el caso anterior sólo el $10,3 \%$ no prevén una mejora de imagen.

Desde la perspectiva externa, otro beneficio asociado a la RSC es el que tiene que ver con la mejora de las relaciones con los agentes del mercado: proveedores, distribuidores y clientes finales.

En el caso de los clientes sólo el 18,2\% de las empresas perciben que la mejora de las relaciones sea altamente improbable. Por el contrario, el 53,6\% de las organizaciones es bastante o totalmente entusiasta a este respecto. Éste es un indicador que señala claramente cómo las empresas analizadas consideran a la RSC un instrumento adecuado en la gestión de sus relaciones con sus clientes.

Cuando el colectivo analizado son los proveedores, se observa una mayor polarización de las respuestas; es decir, aunque el 38,5\% de las empresas no creen que la RSC mejore las relaciones con éstos, el $41 \%$ confían bastante o totalmente en esta expectativa.

Este mismo análisis es extensivo a las relaciones con los distribuidores, es decir, con aquellas otras empresas que adquieren la oferta de las entidades con la finalidad de comerciar con ella. Frente a un $41,1 \%$ de empresas convencidas, el $43,6 \%$ niegan que la RSC lleve asociado este tipo de beneficio. 
De nuevo, y al igual que en los casos anteriores, no se aprecian diferencias significativas en las respuestas en función de las variables de clasificación de la muestra. Las percepciones de los encuestados son homogéneas.

En resumen, del conjunto de factores considerados en relación a los beneficios potenciales asociados a la RSC, que actúan indudablemente como determinantes de su adopción, hemos de reconocer que aquellos vinculados a la proyección externa de las empresas son los que gozan de un mayor grado de aceptación entre las organizaciones analizadas. La RSC es interpretada como una oportunidad para mejorar las relaciones con el entorno, potenciar la imagen y prestigio de las empresas y favorecer sus relaciones comerciales. Las empresas son mucho más reacias a anticipar resultados de naturaleza directamente económica, lo cual parece descartar los intereses puramente economicistas en la implantación de la RSC. Los resultados de tipo interno, especialmente los referidos a la mejora del clima laboral y la adhesión de los trabajadores, gozan de una apreciación intermedia entre las anteriores como consecuencias probables de la RSC.

\subsection{Actividades Socialmente Responsables de tipo Externo o Social}

En este apartado se analiza el empleo por parte de las empresas de la muestra de un conjunto de actividades vinculadas con la aplicación práctica de la RSC en el ámbito externo o social. Lo cierto es que las actuaciones concretas de RSC que desarrollan las organizaciones dependen en gran medida de su propia iniciativa, de sus objetivos estratégicos e incluso, en algunos casos, de determinados procesos regulatorios. Como consecuencia de esta realidad, no existe un único modelo de validez universal que establezca a priori un catálogo general de buenas prácticas, por lo que en este estudio, el conjunto acciones finalmente seleccionadas para su valoración se confecciona a partir de una revisión exhaustiva de la literatura sobre RSC, las normas internacionales en materia de RSC y la consideración de la naturaleza de las organizaciones con las que se desarrolla el trabajo, fundamentalmente de reducido tamaño. Así, el tamaño empresarial, resulta ser una variable muy importante a la hora de mediatizar la implantación de la RSC (Unceta y Gurrutxaga, 2005).

Las actuaciones de ámbito externo seleccionadas ilustran en qué medida la empresa está concienciada de la necesidad de responder responsablemente a las necesidades de su entorno, tanto si éste está representado por sus interlocutores comerciales más directos, clientes o proveedores, como si incluye el conjunto de la sociedad en la que operan las organizaciones. De modo más específico las distintas acciones posibles se clasifican en torno a las relaciones con clientes y proveedores, las políticas de 
información y comunicación a la sociedad y las acciones de colaboración filantrópica que desarrollan las entidades.

La dimensión externa de la RSC constituye, además, el mecanismo más fácilmente enjuiciado por el entorno cuando se trata de establecer la imagen y reputación de las organizaciones, aspecto especialmente valorado por las organizaciones analizadas en el ámbito de los beneficios esperados de la RSC.

\subsection{Relaciones con clientes y proveedores}

En este trabajo el término cliente debe interpretarse en sentido amplio, es decir, agrupa tanto a los distribuidores como a los consumidores de los productos y servicios de la organización. Éstos, junto con los proveedores constituyen los interlocutores comerciales más directos.

La necesidad de relación con estos colectivos viene dada por los objetivos básicos de supervivencia y beneficio de cualquier entidad lucrativa. Sin embargo, desde una perspectiva socialmente responsable, los clientes son los destinatarios últimos de las actuaciones de la empresa y, como tales, los más susceptibles de disfrutar o padecer las consecuencias del consumo de la oferta. La empresa es responsable de sus productos así como del uso e impacto de los mismos a largo plazo, y tiene la obligación de desarrollar relaciones comerciales cuidadosas con los intereses, necesidades y expectativas de servicio de sus clientes. Por su parte, los proveedores ejercen una influencia directa en la cadena de valor de la oferta y en la reputación de la empresa. Su selección adecuada es determinante para la propia acción social de cada entidad, debiéndose asegurar que ofrecen garantías suficientes en cuanto a su comportamiento socialmente responsable.

En base a todas estas consideraciones, las empresas indicaron si ejecutan las acciones propuestas en la Tabla 1. Es reseñable el hecho de que en las relaciones con los clientes y proveedores el $100 \%$ de las empresas analizadas desarrollan alguna de las acciones propuestas, es decir, son activas en las prácticas socialmente responsables de esta naturaleza. Se aprecia que 9 de cada 10 empresas tratan de identificar claramente y con asiduidad las necesidades de sus clientes y que más de 8 de cada 10 tratan de establecer relaciones a largo plazo. Este último aspecto resulta especialmente valioso en los mercados actuales, cada vez más competitivos, en los que la base de compradores no suele crecer, y en los que es más costoso atraer un nuevo cliente que retener a los que ya mantienen una relación comercial con la empresa. En este sentido, casi en 7 de cada 10 empresas existe un sistema regular de atención 
al cliente. El servicio post-venta está disponible, sin embargo, en niveles inferiores a los que serían recomendables, sólo algo más de la mitad de las empresas desarrolla esta actividad.

\section{Tabla I. Relaciones con clientes y proveedores}

\begin{tabular}{|l|c|c|}
\cline { 2 - 3 } \multicolumn{1}{c|}{} & Sí & No \\
\hline $\begin{array}{l}\text { La empresa trata de identificar claramente y con frecuencia las } \\
\text { necesidades de sus clientes }\end{array}$ & $89,5 \%$ & $10,5 \%$ \\
\hline La empresa dispone de un sistema regular de atención al cliente & $68,4 \%$ & $31,6 \%$ \\
\hline Existe un servicio post-venta a disposición del cliente & $52,6 \%$ & $47,4 \%$ \\
\hline $\begin{array}{l}\text { Laempresa busca desarrollar relaciones con sus clientes satisfactorias } \\
\text { a largo plazo para ambas partes }\end{array}$ & $84,2 \%$ & $15,8 \%$ \\
\hline $\begin{array}{l}\text { La empresa desarrolla actividades vinculadas con el comercio } \\
\text { justo }\end{array}$ & $15,8 \%$ & $84,2 \%$ \\
\hline $\begin{array}{l}\text { La empresa está adherida al Sistema Arbitral de Consumo o a } \\
\text { algún otro sistema de mediación o arbitraje }\end{array}$ & $7,9 \%$ & $92,1 \%$ \\
\hline $\begin{array}{l}\text { Laempresa controla que sus proveedores cumplan sus obligaciones } \\
\text { salariales o de seguridad social }\end{array}$ & $15,8 \%$ & $84,2 \%$ \\
\hline $\begin{array}{l}\text { La empresa selecciona a sus proveedores en función de sus prác- } \\
\text { ticas medioambientales }\end{array}$ & $7,9 \%$ & $92,1 \%$ \\
\hline
\end{tabular}

Elaboración propia.

Las iniciativas para promover el comercio justo y la adhesión a sistemas de resolución de conflictos con los clientes son mucho más esporádicas. El comercio justo, no obstante, puede tener repercusiones favorables en determinados clientes especialmente sensibles a las restricciones que infringen los mecanismos producción y distribución de los países más desarrollados al progreso de los países más pobres. De este modo, el comercio justo permite el acceso de la oferta de Economías en desarrollo a los mercados del Primer Mundo y favorecer la distribución de la riqueza; pero para ello se requiere del compromiso de clientes, distribuidores y productores.

En cuanto a los sistemas de resolución de conflictos estos ofrecen una garantía adicional a los clientes de la organización. En concreto, el Sistema Arbitral de Consumo pretende ejercer como mediador resolutivo en aquellas quejas o recla- 
maciones que los consumidores y usuarios puedan efectuar contra los productores o prestadores de servicios.

En las actividades ligadas a las relaciones con clientes, se han encontrado diferencias significativas en tres de ellas:

1. La existencia de un servicio post-venta de atención al cliente. Es más habitual en las empresas cuya actividad principal se ubica en el ámbito de los servicios.

2. El desarrollo de actividades de comercio justo. Mayoritariamente se realiza en las empresas que tienen una dimensión de mercado nacional o internacional.

3. La adhesión de la empresa al sistema arbitral de consumo o a algún otro sistema de mediación o arbitraje. Es más habitual en las cooperativas.

Cualquiera de las iniciativas precedentes responde, además, al convencimiento de que los consumidores exigen cada vez más ofertas cuyo proceso de elaboración sea justo y que, por añadidura, cumplan sus expectativas y respeten su salud y seguridad. Estos comportamientos, por tanto, contribuyen a acrecentar la percepción de la calidad de la oferta, incrementan la fidelidad de los clientes, refuerzan la competitividad de las organizaciones, y permiten que éstas anticipen con mayor facilidad los cambios en los mercados en los que operan. La actuación socialmente responsable con los clientes tiene otra característica destacable y es que se traslada inmediatamente al mercado, cualificando a las empresas para ser seleccionadas como proveedoras de aquellas otras organizaciones que valoren en sus criterios las acciones de RSC.

En cuanto a las relaciones con los proveedores, en el cuestionario se valora en qué medida las empresas se preocupan por su cumplimiento con las obligaciones salariales y de seguridad social que les atañen, así como su nivel de compromiso con el respeto al medioambiente. Los resultados de este análisis, mostrados en la Tabla 1, evidencian que tanto en un caso como en otro las empresas de la muestra se abstienen mayoritariamente de desarrollar este tipo de control.

No obstante, seleccionar a proveedores socialmente responsables lleva asociadas una serie de ventajas derivadas. Estos proveedores es más probable que sean más conscientes de la necesidad de generar valor a sus clientes, por lo que tratándose de relaciones entre empresas, es más esperable que contribuyan a la mejora de su productividad, eficiencia y valor añadido. Las relaciones con proveedores comprometidos también abren puertas para una mayor innovación en los productos y 
servicios de la empresa-sugerida por estos-, la identificación de nuevos clientes o mercados potenciales, o el aumento de los servicios periféricos o complementarios a la oferta básica que ofrecen las organizaciones.

\subsubsection{Procedimientos de información y comunicación a la sociedad}

Tan importante como que las empresas desarrollen acciones socialmente responsables es que logren transmitir a la sociedad esta circunstancia. Además, el esfuerzo de comunicación debe iniciarse en el etiquetado, envasado o información elaborada por la empresa que acompañe a su oferta. Los contenidos ofrecidos a los clientes deben cumplir la legislación vigente que les afecte. Así mismo, la comunicación masiva sobre la empresa y sus productos y/o servicios también está sujeta a regulación, por lo que como mínimo debe ser veraz y respetuosa.

En consonancia con todas estas observaciones, el cuestionario incluyó las variables recogidas en la Tabla 2. La interpretación de los resultados en este punto debe efectuarse teniendo en cuenta que algo más de la mitad de las empresas de la muestra son inactivas en esta materia. Este dato se puede justificar teniendo en cuenta que las políticas de comunicación empresarial suelen ser bastante deficientes en la inmensa mayoría de las PYME, las cuales no cuentan generalmente con estrategias claras en la materia o con una asignación de recursos consistente. Por tanto, las empresas que no desarrollan acciones de comunicación habitualmente es probable que no hagan publicidad o que no se preocupen de transmitir su comportamiento socialmente responsable. Es cierto, no obstante, que la no atención a las exigencias de información sobre la oferta es menos excusable.

\section{Tabla 2. Procedimientos de información y comunicación a la sociedad}

\begin{tabular}{|l|c|c|}
\cline { 2 - 3 } \multicolumn{1}{c|}{} & Si & No \\
\hline $\begin{array}{l}\text { La empresa cumple con todos los requisitos acerca del envasado, } \\
\text { etiquetado, empaquetado o información sobre sus productos }\end{array}$ & $66,7 \%$ & $33,3 \%$ \\
\hline $\begin{array}{l}\text { La empresa ha establecido mecanismos para que el contenido de } \\
\text { su publicidad sea veraz, transparente y respetuoso }\end{array}$ & $88,9 \%$ & $11,1 \%$ \\
\hline $\begin{array}{l}\text { La empresa publica información sobre acciones de RSC } \\
\text { anualmente }\end{array}$ & - & $100 \%$ \\
\hline
\end{tabular}

Elaboración propia. 
Tal y como recoge la Tabla 2 , casi 7 de cada 10 empresas cumplen todos los requisitos sobre envasado, etiquetado, empaquetado o información sobre sus productos. El porcentaje de organizaciones que, además, practica una política de transparencia publicitaria asciende al $90 \%$. Este es un dato muy positivo puesto que la utilización fraudulenta de este instrumento de comunicación, aunque perseguida legalmente, sigue produciéndose en muchos casos. No obstante, ninguna de las organizaciones analizadas comunica sus acciones de RSC. Creemos que es preciso insistir en que las políticas informativas suelen estar muy descuidadas en las empresas más pequeñas, las cuales no suelen contar con profesionales cualificados en este campo ni con los recursos necesarios para desarrollarlas consistentemente. En este caso no se han encontrado diferencias significativas en función de las variables de clasificación en el comportamiento de las empresas de la muestra.

\subsubsection{Fórmulas de colaboración responsable}

Las empresas no pueden conformarse con la creencia de que mediante la generación de empleo y de riqueza se justifica su contribución a la mejora de la sociedad. El respeto al medioambiente nace como consecuencia de este principio y se completa con el apoyo de necesidades sociales concretas. Uno de los enfoques más novedosos en este campo es la inversión responsable, que persigue una utilización equitativa y socialmente consecuente de los fondos obtenidos. La inversión responsable tiene dos vertientes. Por un lado, consiste en investigar a fondo los lados positivos y negativos de las empresas desechando aquellas cuyas prácticas son percibidas como dañinas para la gente o el medio ambiente; es decir, se trata de dirigir la inversión empresarial hacia aquellas organizaciones que ofrezcan ingresos sólidos pero que también sean líderes en las prestaciones sociales y ambientales (Camprodón, Sols y Florensa, 2006). Otro enfoque consiste en organizar el poder de los accionistas/propietarios para presionar activamente la toma de decisiones socialmente responsables ${ }^{8}$.

No obstante, la inversión responsable es muy escasa en nuestro país, de acuerdo con los últimos estudios disponibles, y sobre todo en el ámbito de las PYME?. Sin

\footnotetext{
${ }^{8}$ La presión de los accionistas, por ejemplo, convenció a la empresa Mitsubishi a abandonar sus planes de fundar una salina en el golfo de California (México) lo cual hubiera destruido una zona de reproducción para las ballenas grises. La inversión responsable socialmente en otra época se consideraba solamente un medio para hacer el bien, pero hoy, sus partidarios insisten en que tan sólo es una inversión inteligente.
}

${ }^{9}$ La inversión socialmente responsable no parece conseguir captar la atención de los españoles ni recibe el impulso de bancos y gestores de inversión. El patrimonio gestionado en este tipo de fondos supone 
embargo, las empresas pueden optar por otra alternativa de actuación mucho más accesible y popularizada hasta la fecha; nos referimos a la colaboración con distintos proyectos sociales, ya sea con instituciones comunitarias, municipales y gubernamentales, u organizaciones no gubernamentales (ONGs). De hecho, la colaboración de la empresa con la comunidad ha sido desde siempre parte de la RSC, y especialmente en el caso de las PYME, para las que constituye la opción práctica más accesible. La colaboración puede materializarse en forma de aportaciones económicas a las distintas causas sociales o mediante la participación de los empleados o productos de la empresa en las mismas. Además, lo ideal es que el apoyo a las causas sociales se complemente con un impacto positivo en la estrategia de negocio. Es decir, las empresas no pueden regirse exclusivamente por criterios filantrópicos y deben buscar una cierta coherencia entre el tipo de actuaciones apoyadas, su tipo de actividad y el perfil del público al que se dirigen.

\section{Tabla 3. Fórmulas de colaboración responsable}

\begin{tabular}{|c|c|c|}
\hline & Sí & No \\
\hline $\begin{array}{l}\text { La empresa colabora habitualmente con ONG's, Fundaciones o } \\
\text { Entidades sin ánimo de lucro }\end{array}$ & $59,1 \%$ & $40,9 \%$ \\
\hline La empresa apoya el desarrollo de actividades deportivas & $27,3 \%$ & $72,7 \%$ \\
\hline $\begin{array}{l}\text { La empresa apoya el desarrollo de actividades culturales y } \\
\text { educativas }\end{array}$ & $45,5 \%$ & $54,5 \%$ \\
\hline $\begin{array}{l}\text { La empresa apoya actividades dirigidas a grupos } \\
\text { desfavorecidos }\end{array}$ & $18,2 \%$ & $81,8 \%$ \\
\hline $\begin{array}{l}\text { La empresa apoya actividades medioambientales no ligadas a } \\
\text { su propia actividad }\end{array}$ & $4,5 \%$ & $95,5 \%$ \\
\hline $\begin{array}{l}\text { La empresa facilita la participación de sus empleados en } \\
\text { actividades socialmente responsables }\end{array}$ & $18,2 \%$ & $81,8 \%$ \\
\hline La empresa efectúa donaciones no económicas & $18,2 \%$ & $81,8 \%$ \\
\hline
\end{tabular}

Elaboración propia.

La Tabla 3 muestra las distintas iniciativas recogidas en este apartado. En este caso es necesario poner de manifiesto que el $41 \%$ de las empresas de la muestra son

sólo 1.168 millones de euros, el 0,4\% del total. Sus activos crecieron en 143 millones de euros (tres décimas) en 2006, según la Comisión Nacional del Mercado de Valores, una cifra insignificante frente al aumento del $27 \%$ en el patrimonio obtenido por este tipo de fondos en el conjunto de Europa. 
inactivas, no desarrollan fórmulas de colaboración responsable. No obstante, entre las empresas activas casi 6 de cada 10 colaboran habitualmente con entidades sin ánimo de lucro, lo que constituye un porcentaje especialmente notable dado el reducido tamaño y volumen de la mayor parte de las organizaciones de la muestra. Sin duda, éste es un dato muy ilustrativo del compromiso social de este colectivo. Las actividades fundamentalmente apoyadas son de naturaleza cultural y educativa $(45,5 \%)$, seguidas de las de tipo deportivo $(27,3 \%)$.

Sin embargo, otro tipo de iniciativas, como las dirigidas a grupos desfavorecidos, la realización de donaciones no económicas o la participación de los empleados facilitada por la empresa en actividades socialmente responsables están presentes en menor medida, aproximadamente en un $20 \%$ de las empresas analizadas. Así mismo, se constata que el apoyo de actividades medioambientales no vinculadas a la propia actividad de las empresas tiene una presencia marginal.

Del conjunto de acciones que constituyen las fórmulas de colaboración responsable, hemos encontrado diferencias significativas en una de ellas, el apoyo de actividades dirigidas a grupos desfavorecidos. Esta acción es desarrollada principalmente por cooperativas y por las empresas más pequeñas.

\section{5. ¿Regulación de la RSC?}

Un debate que ha suscitado considerable interés en los últimos años es si el desarrollo de la RSC debería de estar regulado o debería de dejarse a iniciativa de cada empresa como una opción voluntaria que compete al ámbito del desarrollo de sus operaciones. Generalmente, la respuesta a esta pregunta por parte del mundo empresarial es que el compromiso de las empresas con la RSC no debería de ser regulado, partiendo de la base de que el propio concepto alude a lo que las empresas hacen más allá de sus obligaciones legales.

Sin embargo existen ciertos fallos de mercado y otras cuestiones de carácter social o ético que hacen necesaria una intervención pública dirigida al fomento, promoción, y sensibilización social para aumentar la información y la transparencia en el mercado. Además, algunos aspectos relacionados con la RSC no son sólo de carácter privado sino que entran en el terreno de lo público. Entre otros, cabe mencionar la sanidad, el deterioro medioambiental, el efecto dañino de ciertos productos e incluso algunas políticas monetarias, fiscales y de comercio internacional.

El Informe final de la "Subcomisión del Congreso de los Diputados para potenciar y promover la Responsabilidad Social de las empresas" concluye que los compa- 
recientes ante la comisión mantuvieron diferencias de criterio sobre el alcance y profundidad deseables de las iniciativas reguladoras en materia de RSC por parte de las Administraciones Públicas, aunque establece algunas recomendaciones que pueden desempeñar un papel regulador: el desarrollo de una normativa que obligue a las entidades gestoras de fondos de inversión y planes de pensiones a indicar si incorporan o no criterios sociales y ambientales en la selección de inversiones; la promoción de reducciones fiscales para los inversores que inviertan en fondos específicamente destinados a financiar actividades económicas beneficiosas para el medio ambiente; o bonificaciones en la Seguridad Social como instrumento de integración o la promoción de un marco fiscal favorable para las empresas, entre otras. También se señala como instrumento de promoción de la RSC los sistemas de preferencia en las compras públicas, que favorezcan la implantación de la RSC en las PYME, y medidas en favor de aquellas empresas transnacionales que contribuyan a exportar prácticas socialmente responsables a países con legislaciones más laxas que la española y comunitaria.

Para las CC.AA., por su parte, la promoción de la RSC tiene una lógica políticoeconómica clara: si las empresas que practican RSC son más competitivas, las regiones que tengan empresas de este tipo también lo serán. Además, la RSC no sólo mejora la imagen y la respetabilidad de las empresas, sino también la imagen y la respetabilidad de la región y promueve su desarrollo económico, social y medioambiental sostenible.

Quizá el futuro pase por concebir la responsabilidad social como un terreno compartido donde la iniciativa pública asuma el compromiso de establecer un marco coherente para la actuación de los agentes privados y dotar de transversalidad a unas iniciativas que por su propia naturaleza están llamadas a afectar a múltiples interesados (ANCOS, 2007).

Sin embargo, los resultados de este estudio indican que para las empresas la implementación de la RSC parece que debe dejarse a la autorregulación. La mayoría de las empresas encuestadas se muestran a favor de la voluntariedad de la RSC. Así, más de un 50\% de los encuestados declaran que no creen que la RSC deba ser regulada, pero que las Administraciones Públicas sí deberían recomendarla y fomentarla con ayudas. Un $20 \%$ cree además que aunque no debería ser regulada, debería ser también fomentada por las Asociaciones Empresariales (Tabla 4). Tan sólo el 7,69\% de las empresas creen que la RSC debería regularse legislativamente con normativas de carácter nacional y casi el $18 \%$ que debería de regularse con normativas de carácter internacional. Estos resultados son de nuevo consistentes para el conjunto de entidades analizadas. 
Tabla 4. Necesidad de regulación de la RSC

\begin{tabular}{|l|c|c|}
\cline { 2 - 3 } \multicolumn{1}{c|}{} & Sí & No \\
\hline Debe dejarse a la iniciativa de cada empresa y no regularse & $15,38 \%$ & $84,62 \%$ \\
\hline $\begin{array}{l}\text { No debe regularse, aunque las Administraciones Públicas deberían } \\
\text { recomendarla y fomentarla con ayudas. }\end{array}$ & $53,85 \%$ & $46,15 \%$ \\
\hline $\begin{array}{l}\text { No debe regularse, aunque las Asociaciones Empresariales de- } \\
\text { berían recomendarla y fomentarla }\end{array}$ & $20,51 \%$ & $79,49 \%$ \\
\hline $\begin{array}{l}\text { Debe regularse legislativamente con normativas de carácter } \\
\text { nacional }\end{array}$ & $7,69 \%$ & $92,31 \%$ \\
\hline Debe regularse legislativamente a nivel de la Unión Europea & $17,95 \%$ & $82,05 \%$ \\
\hline
\end{tabular}

Elaboración propia.

\section{Conclusiones}

Parece claro que en la actualidad las empresas son cada vez más conscientes de que no pueden actuar como islas en la sociedad centradas en la maximización de beneficios sino que la propia sociedad les reclama una contribución activa al desarrollo colectivo. De este modo la gestión socialmente responsable se ha convertido en el concepto de moda tanto en las organizaciones empresariales como en los círculos académicos (ABAD, 2005). La RSC incluye las acciones voluntarias de las empresas dirigidas a atender las preocupaciones de trabajadores, clientes, proveedores, inversores $y$ /o accionistas $y$, en general, del conjunto de comunidad en la que operan. Así, la RSC responde a un movimiento de demanda por parte de la sociedad civil, a la que los Estados no han sido ajenos, regulando distintos aspectos laborales, medioambientales y vinculados con la transparencia y con la protección de consumidores y usuarios. No obstante, la definición de RSC reflejada en este estudio implica que más allá de esta regulación, las prácticas socialmente responsables deben ser fruto del compromiso de las empresas con el desarrollo de sociedades más equitativas y respetuosas con su entorno.

Frecuentemente al hablar de RSC la referencia son las grandes empresas, sin embargo, en la Unión Europea las PYME representan la mayor parte del tejido empresarial, siendo su papel económico fundamental. Las PYME encuentran muchas más dificultades para la implantación de la RSC dado su menor tamaño y falta de 
recursos económicos y humanos, pero ello no implica que la RSC no pueda derivar en los mismos beneficios que los que potencialmente se pueden obtener en las organizaciones más grandes.

La dificultad de implantación de la RSC en las PYME es reconocida por la Administración Pública, la cual, a través de diversos informes de expertos, reconoce la necesidad de un apoyo decidido a este tipo de empresas tanto en materia informativa como para ayudarles a implementar buenas prácticas. Sin embargo, es aún largo el camino que queda por recorrer, siendo necesario un mayor número de estudios que analicen la percepción que tienen las empresas sobre la RSC, cómo se desarrolla su puesta en práctica y las medidas adecuadas para el fomento y mayor conocimiento de la RSC por parte de las empresas. Es en este ámbito en el que se enmarca este estudio. Por añadidura, al analizar un tipo de figuras empresariales, las empresas de Economía Social, que se acomodan muy bien a los planteamientos de las RSC por los valores y principios que rigen su funcionamiento, tratamos de contribuir a la competitividad de un tipo de empresas que han visto acrecentado su ámbito de actuación y su dimensión empresarial, por lo que deben competir en igualdad de condiciones con el resto de organizaciones.

El análisis de las motivaciones o beneficios que las organizaciones esperan en la aplicación de la RSC revela que, en el plano económico, el aspecto más valorado es la obtención de incentivos fiscales asociados a ciertas actuaciones, como las donaciones a entidades no lucrativas. Resulta destacable que se otorga escasa importancia a la obtención directa de beneficios económicos. Entre las posibles motivaciones internas para desarrollar la RSC en las empresas prevalece la mejora del clima laboral. Y entre las motivaciones externas, las más importantes en conjunto, se destaca la mejora de la imagen y prestigio de la empresa seguido de la mejora de la relación con los clientes y con los proveedores.

Las actividades concretas de RSC de tipo externo o social incluyen las relaciones con clientes y proveedores, los procedimientos de información y comunicación a la sociedad, y las fórmulas de colaboración responsable. El 100\% de las empresas son activas en lo que se refiere a las relaciones con los agentes del mercado, clientes y proveedores. Sin embargo, las iniciativas más frecuentes se dirigen a los clientes: identificación de sus necesidades, atención en el servicio post venta y el desarrollo de relaciones a largo plazo. El menor seguimiento de las prácticas sociales de los proveedores puede justificarse en el tamaño y juventud de las empresas analizadas, lo cual dificulta su poder de negociación y control en el mercado. Los procedimientos de comunicación e información a la sociedad tienen lugar en aproximadamente la mitad de las empresas. Constituyen, por tanto, el tipo de prácticas de RSC menos 
frecuente en la muestra, lo que resulta coherente en el contexto de escaso desarrollo de la política de comunicación que caracteriza a muchas PYME. La colaboración responsable, sin embargo, se lleva a cabo en más del $60 \%$ de las empresas analizadas destacando la involucración con entidades sin ánimo de lucro y su apoyo a actividades de naturaleza cultural y deportiva.

Los resultados del estudio también revelan que las PYME analizadas que valoran en mayor número y medida los posibles beneficios asociados a la RSC son las que también aplican más prácticas en este ámbito. No obstante, los encuestados insisten en que la implantación de la RSC debe llevarse a cabo desde el ámbito de la autorregulación, sin que ello excluya la labor promotora en forma de ayudas e información, de las Administraciones Públicas y asociaciones empresariales.

En definitiva, las empresas de Economía Social analizadas ponen de manifiesto una indudable vocación de comportamiento socialmente responsable en el ámbito externo o social de sus operaciones, que es efectivamente llevada a la práctica con las lógicas limitaciones derivadas de su menor tamaño y recursos. No parecen guiadas exclusivamente por intereses economicistas, sino que valoran en especial la mejora del clima interno y la imagen que transmiten a la sociedad en la que operan.

En este sentido la literatura advierte que la RSC ofrece a las empresas de Economía Social la posibilidad de construir un posicionamiento diferenciado basado en su mayor compromiso con la comunidad, el cual se ve reforzado por la "marca social" que diferencia a estas organizaciones de las empresas de capitales. No obstante, y dado que la RSC no está libre del estigma de ser considerada una mera operación cosmética, mientras que las organizaciones de capitales deben acreditar que sus prácticas de RSC son algo más que un medio para alcanzar sus objetivos, las empresas de Economía Social deben saber transmitir a la sociedad que las prácticas de RSC son un fin y no un medio.

Consciente de esta importancia y de sus potenciales ventajas, el sector de la Economía Social está impulsando decididamente la aplicación de la RSC entre sus empresas, como lo demuestra la reciente puesta en marcha del Grupo Europeo para el Balance Social (GEBS) de las empresas de Economía Social, como plataforma para su evaluación continua.

Adoptando una perspectiva de futuro, uno de los retos pendientes en materia de RSC, y al que no son ajenas las empresas de Economía Social, es lograr la integración estratégica de las actividades sociales. Así, la acción social alcanza su 
madurez cuando se plantea como una actividad estratégica y, por tanto, difícil de separar de la actividad económica, transversal a todas sus áreas, con proyección a medio plazo y apoyada por los distintos tipos de recursos organizativos (ABAD, 2005). No podemos ser ajenos al hecho de que desarrollar de manera informal o no premeditada las prácticas socialmente responsables no es una vía sostenible a medio plazo (SANCHís y CAMPOS, 2007). De este modo, las empresas de Economía Social deben ver en el desarrollo estratégico de la RSC una fuente de ventaja competitiva especialmente valiosa en los mercados actuales y que, al mismo tiempo, guarda una gran concomitancia con los principios que rigen estas organizaciones. En las empresas de Economía Social existe así a priori un "ajuste" cultural que debe explotarse adecuadamente dotando a las prácticas de responsabilidad social de carácter estratégico. Las PYME, categoría a la que pertenecen en su mayor parte las organizaciones de Economía Social, deben ser especialmente proactivas en la identificación y explotación de sus recursos valiosos, para compensar otras carencias derivadas de su menor tamaño empresarial, lo que hace que desde esta perspectiva la trascendencia de la estrategia de RSC sea aún mayor.

\section{Bibliografía}

Abad Jiménez. F. (2005) "¿Políticas públicas sobre acción social empresarial?", CIRIEC-España, Revista de Economía Pública, Social y Cooperativa, n 53, noviembre, pp. 19-28.

Ancos Franco. H. (2007) "Políticas públicas e iniciativa privada en la responsabilidad social empresarial", Revista del Ministerio de Trabajo y Asuntos Sociales, $\mathrm{n}^{\circ}$ 66, pp. 51-79.

BOWEN, H. (1953) Social Responsibilities of the Businessman, Nueva York: Harper and Brothers.

Camprodon Rosanas, M.; Sols Lucia, J., Florensa Giménez, A. (2006) “Las agencias de evaluación de la Responsabilidad Social Corporativa: estudio de un caso", Revista de Fomento Social, $n^{\circ} 243$, julio-septiembre, pp. 393-422

CANALS, J. y Fontrodona, J. (2006) "Responsabilidad Corporativa y Gobierno de la Empresa", en Olcese y otros (ed.): La Responsabilidad Corporativa: Una propuesta para un entorno empresarial más eficiente y socialmente comprometido. Fundación de Estudios Financieros, Papeles de la Fundación, n 16, pp. 35-52. 
Castro Sanz, M. (2005) "La Responsabilidad Social de las Empresas, o un nuevo concepto de empresa", CIRIEC-España. Revista de economía pública, social y Cooperativa, $n^{\circ} 53$, pp. 29-51.

Castro SAnz, M. (2006: "Las empresas de economía social y la responsabilidad social corporativa", Papeles de Economía Española, n 108, pp. 92-105.

Collado Conde, J. L. (2006) "La Responsabilidad Social Corporativa y las Cooperativas: Aspectos y Formulaciones Comunes". XI Jornadas de Investigadores en Economía Social y Cooperativa. Santiago de Compostela.

Consejo de RedACCIÓN (2006) "La responsabilidad social de la empresa: ¿el coste de tener conciencia?", Revista de Fomento Social, n 244, octubre-diciembre, pp. 499-517.

Directorio Central de Empresas (DIRCE) Instituto Nacional de Estadística, http://www. ine.es/ (15/01/2008)

ElkINGTON, J. (1997) "Chapter 6-Values: Ghost in the System". Cannibals with Forks: The Triple Bottom Line of 21 st Century Business, Capstone Publishing Limited.

European Foundation for Qualty Management (2007) hitp://www.efqm.org/ (31/12/2007).

Foro de Expertos en Responsabilidad Social de las Empresas, Informe Final (2007) http://www.mtas.es/Empleo/economia-soc/RespoSocEmpresas/indiceRespo.htm (15/01/2008).

Ffes (2003) Fundación para el Fomento de la Economía Social. El Libro Blanco de la Economía Social en el Principado de Asturias.

Global RePORTING INITIATIVE (http://www.globalreporting.org/)

LBROVERDELACOMISIÓNEUROPEA(2001)http://ec.europa.eu/employment_social/soc-dial/ csr/greenpaper_es.pdf.

Millán de las Heras, J. L. y Berin Pérez, M. (2006) "Cooperativas y Responsabilidad Social". XI Jornadas de Investigadores en Economía Social y Cooperativa. Santiago de Compostela. 
Montero, M. J., Araque, R. y Rey, J. M. (2007) "La seguridad y salud en el trabajo en el marco de la responsabilidad social de la empresa", Revista de Fomento Social, $n^{\circ}$ 246, abril-junio, pp. 199-216.

OCDE (2000) Guidelines for Multinational Enterprises (http://www.oecd.org/)

OlCESE, A. (2006) "Introducción y conclusiones", en Olcese y otros (ed.): La Responsabilidad Corporativa. Una propuesta para un entorno empresarial más eficiente y socialmente comprometido. Fundación de Estudios Financieros, Papeles de la Fundación, $n^{\circ} 16$, pp. 11-33.

PACTO MUndial (2000) http://www.pactomundial.org/ (15/01/2008)

Pino Artacho, J. (2003) "Responsabilidad de las empresas de economía social". Abaco: Revista de cultura y ciencias sociales, $n^{\circ} 36, \mathrm{pp} .43-48$.

Salas, J. y García-Orcoyen, C. (2006) "RSE y Desarrollo Sostenibles", en Olcese y otros (ed.): La Responsabilidad Corporativa: Una propuesta para un entorno empresarial más eficiente y socialmente comprometido. Fundación de Estudios Financieros, Papeles de la Fundación, n 16, pp. 72-87.

Sanchís Palacio, J. R. y Campos Climent, V. (2007) "La dirección estratégica en la de economía social: utilización de herramientas de análisis estratégicos en las cooperativas", CIRIEC-España, Revista de Economía Pública, Social y Cooperativa, $\mathrm{n}^{\circ} 59$, octubre, pp. 237-258.

Unceta Satrústegul, A. y GurRutXaga Abad, A. (2005) Responsabilidad Social Corporativa en el País Vasco. Consejería de Industria, comercio y Turismo. Gobierno Vasco.

VÁzQuez GarcíA, M. (2006) "Responsabilidad social de la PYME", Boletín Económico de ICE, $\mathrm{n}^{\circ} 2901$, pp. 31-34.

Vives, A.; Corral, A. e IsasI, I. (2005) Responsabilidad Social de la Empresa en las PYMEs de Latinoamérica. Banco Interamericano de Desarrollo: www.iadb.org (15/01/2008).

Wood, D. J. (1991) "Corporate Social Performance Revisited". Academy of Management Review, Vol. 16; n 4, pp. 691-718. 